BBA 45552

\title{
DICHROISM IN SPINACH CHLOROPLASTS
}

\author{
J. B. THOMAS, J. H. VAN LIEROP AND M. TEN HAM
}

Biophysical Research Group, Physics Institute, State University, Utrecht (The Netherlands)

(Received November 8th, I966)

\section{SUMMARY}

In spinach chloroplasts oriented at steel-water interfaces parallel to the light beam a distinct dichroism is measured at about $680 \mathrm{~nm}$. This dichroism is minimal upon addition of sucrose up to a final concentration of $0.18 \mathrm{M}$ to the medium, the dichroic ratio amounting to $\mathrm{x} .02$. It is concluded that predominantly the $\mathrm{C}_{\boldsymbol{a}} 68 \mathrm{O}$ chlorophyll type is oriented to an extent of about $2 \%$ in the plane of the chloroplast lamellae.

No indication could be obtained that the $C_{a} 680$ molecules occur at an angle other than $0^{\circ}$ or $90^{\circ}$ to the surface of the quantasome subunits. For reasons mentioned in DIscussion, it is suggested that a tangential arrangement of these molecules at the subunit surfaces seems to be more likely than a radial one.

Based on the present experiments as well as on data from the literature a hypothetical model for the arrangement of the photosynthetic pigments in the quantasome is given.

\section{INTRODUCTION}

The orientation of chlorophyll molecules in the chloroplast has been studied by various authors ${ }^{1-14}$ and in various manners. However, the interpretations of the results are contradictory. Some investigators ${ }^{2,5}$ are of the opinion that the observed phenomena should be attributed to textural anisotropy rather than to orientation of the pigment molecules, whilst no agreement about the identity of the possibly oriented chlorophyll a form exists (cf. refs. Io-13).

The problem of chlorophyll orientation is of interest with regard to energy transfer ( $c f$. ref. I5), the solid state conception of photosynthesis ${ }^{16-19}$, and the molecular structure of the photosynthetic apparatus. Electron-microscopical studies ${ }^{20-22}$ suggest that the interfaces to which the pigment molecules may be oriented are not flat. Moreover, both submicroscopical and analytical data should be taken into consideration when studying chlorophyll orientation.

It therefore seemed worth while to check, with a different, rather simple technique, to what extent chloroplast dichroism is due to orientation of pigment molecules, in what way such an arrangement may occur, and which types of chlorophyll complexes may be engaged, as well as to try to fit these data into a picture in which the various data from the literature are incorporated.

Abbreviation: DCIP, 2,6-dichlorophenolindophenol. 
METHODS

\section{Material}

Spinach leaves were obtained either commercially or from the botanical gardens. The leaves were depetioled, thoroughly rinsed with tap-water, and finally washed in o. I5 M phosphate buffer of $\mathrm{pH} 6.7$. This buffer was used throughout the experiments. The following manipulations were carried out in the cold and in as dim light as possible.

About $400 \mathrm{~g}$ of leaves were minced in a Braun multipress provided with a strip of filter paper along the perforated wall of the rotating trough. During mincing, buffer was added, and the suspension was collected in a flask already containing some buffer. The resulting suspension was diluted with roughly the same amount of buffer and passed through filter paper once more. For each series of measurements a fresh preparation was made. Just before use, the suspension was diluted to such a concentration that absorption in a $\mathrm{I}-\mathrm{cm}$ pathlength amounted to about $70 \%$ at the red maximum.

\section{Orienting the chloroplasts}

The chloroplasts were oriented as described earlier ${ }^{23}$. Since the procedure was slightly changed, a brief description may follow here. Fig. I shows the arrangement. Two dry identical piles of $10020 \mathrm{~mm} \times 20 \mathrm{~mm} \times 0.18 \mathrm{~mm}$ polished stainless-steel platelets (C), kept apart by $0.04-\mathrm{mm}$ thick aluminium spacer strips (D) on two opposite sides, were loaded with chloroplasts by immersing the piles in a chloroplast suspension and evacuating for about $20 \mathrm{~min}$. Next, by cautiously admitting air, chloroplast suspension was allowed to enter in between the platelets (C). Since spinach chloroplasts are relatively flat, they will arrange themselves parallel to the platelet surfaces by either sedimenting, or, if their boundary is hydrophobic, adhering to both the upper and lower steel surfaces. The thus-loaded piles were placed in perspex holders (E) containing a few $\mathrm{ml}$ of chloroplast suspension. In prolonged experiments it

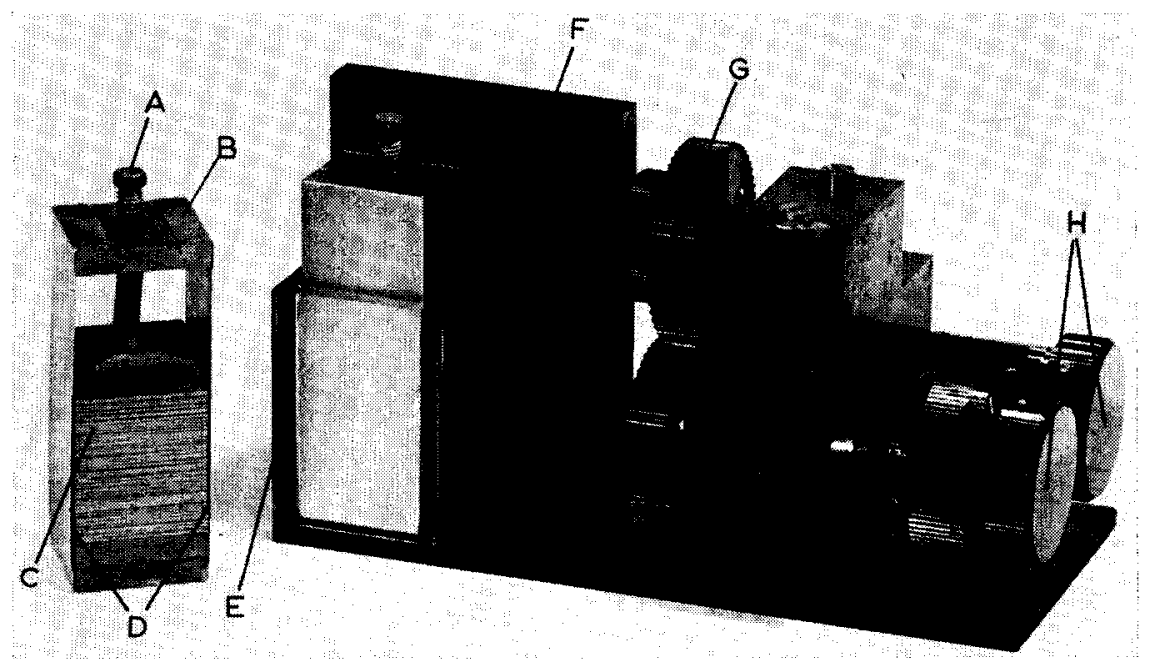

Fig. I. Pile and arrangement for dichroism measurements. A, screw for rigidly clamping the stainless-steel platelets C; B, brass block; D, positions of aluminium spacer strips; E, perspex holder; F, black plastic wall separating the two piles; $G$, gear-wheel for rotating both polaroids simultaneously; $\mathrm{H}$, polaroids adjusted perpendicularly to each other. 
proved necessary to avoid settling of the chloroplasts suspended in between the holder walls and the piles. In this case the loaded piles were dipped first in buffer to remove chloroplasts adhering to the sides, and next placed into the holders containing a few $\mathrm{ml}$ of buffer. Care was taken that no air bubbles were enclosed when pressing the piles into the holders. The fact that within a few minutes after loading the piles a dichroic effect could be reproducibly measured demonstrates that arrangement of the chloroplasts occurred nearly instantaneously. However, from the experiments on the effect of rotation of the polaroid system, which lasted for more than $\mathbf{I} h$, it became evident that, after some time, the dichroic effect started to decline slowly until, finally, a somewhat lower value was reached, the periods and degree of decrease varying for different preparations. This inconvenience was overcome by storing the entire set-up in the dark at $0^{\circ}$ for I-I.5 h before starting the "rotation" experiments, provided no sucrose was added. In the latter case, both the steepness and the duration of the decline increased, whereas the reproducibility of the measurements declined. Therefore, the pre-storage period with the latter experiments was not prolonged beyond $20 \mathrm{~min}$.

\section{Measurement of dichroism}

The apparatus shown in Fig. I was placed with precision in the cuvette compartment of a Beckman DK 2 recording spectrophotometer, the piles facing the windows of the photomultiplier compartment. Both beams entering the piles were polarized in mutually perpendicular planes. By turning the gear-wheel G, Fig. I, both polaroids could be rotated simultaneously, their polarization planes remaining perpendicular to one another. If the beams in the reference channel and sample channel were polarized horizontally and vertically respectively, the polaroids are said to be rotated $0^{\circ}$ or $360^{\circ}$. Turning the polaroids occurred in a clock-wise manner. In some of the experiments fixed polaroids were used as described earlier ${ }^{23}$.

In this way a dichroic effect is recorded directly as a difference spectrum. Control experiments have been described in the earlier paper ${ }^{23}$ and will not therefore be considered here.

In most cases, the light transmission was slightly different for each pile. For the major part, this difference was due to a few air bubbles remaining in between the platelets after evacuation. Two inconvenient effects are caused by these air bubbles, namely unbalance of (I) scattering and reflecting properties of the two piles, and (2) light-paths through the two chloroplast preparations. The first effect was corrected for by using an adjustable light screen, whereas the second one was taken care of by placing a $I-\mathrm{cm}$ glass cuvette in front of each pile, one of them containing buffer solution, the other one, in front of the pile with the deficit of chloroplasts, filled with a very dilute chloroplast suspension so as to compensate for the absorption difference in non-polarized light. As this procedure is time-consuming it could not be applied when performing a relatively large number of subsequent experiments. In the latter case a correction factor was experimentally established for the various deviations from the zero line of the difference spectrum in a region of negligible absorption, $750 \mathrm{~nm}$, whereas the intensity of the band around $680 \mathrm{~nm}$ in this spectrum was corrected for with that at the $45^{\circ}$ position of the polaroids. Since check experiments ${ }^{23}$ proved that at this position of the polaroids any dichroic effect was absent, such a procedure is permissible. 


\section{RESULTS}

\section{Nature of the dichroic effect}

An example of the dichroic effect in oriented spinach chloroplasts, corrected for scattering, reflection and light-path anomalies, is shown in Fig. 2. This effect, representing the difference in percent transmission of both chloroplast-loaded piles, is measured by the distance between band maximum and base, the latter being drawn as a straight line through the recorded base on the longwave side. As a rule, the dichroic effect is lower than that depicted in Fig. 2. Out of I4 experiments an average of $8.0 \pm 0.6 \%$ was calculated.

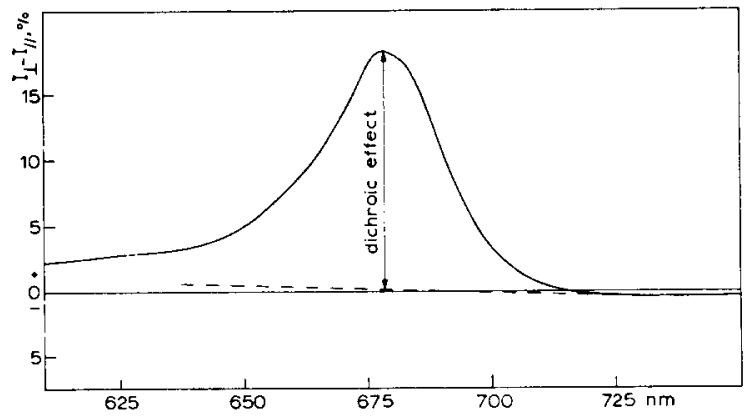

Fig. 2. Example of the dichroic effect in oriented spinach chloroplasts. Along the ordinate transmission differences are plotted.

The dichroic effect invariably shows up in chloroplasts obtained from spinach grown in spring, summer and early autumn. Towards winter, the dichroism decreases until it is completely absent in mid-winter. Figs. $3 \mathrm{~A}$ and $3 \mathrm{~B}$ may demonstrate why in winter time no dichroism is observed. Fig. $3 \mathrm{~A}$ shows an in situ chloroplast typical of summer spinach, whereas in Fig. ${ }_{3} \mathrm{~B}$ such a chloroplast of mid-winter spinach is depicted. For both batches the same kind of seed was used, while no additional illumination was given. From the electron micrographs it is clear that a dichroic effect can be anticipated only in summer for two reasons: (I) summer chloroplasts are flat and lens-shaped and, thus, easy to orient on surfaces, whereas the winter chloroplasts are nearly spherical, and (2) as a rule the lamellar structures run parallel to the flat outer layers of the summer chloroplasts, while they are twisted in all directions in winter chloroplasts.

It is additionally remarked that the absorption spectra are not notably different for summer and winter chloroplasts. Fluorescence spectra of only three preparations of each type of chloroplast were recorded. This limited number of experiments seems to suggest that at room temperature no difference occurs, whereas at - I96 $6^{\circ}$ (cf. Fig. 4) a faint shoulder around $684 \mathrm{~nm}$ observed with summer chloroplasts shows up as a distinct maximum at $685 \mathrm{~nm}$ in winter chloroplasts. Such an effect may indicate that in winter plastids, energy transfer from the F-685 to the F-695 chlorophyll component is considerably reduced. The same is true of the capacity for the Hill reaction, with $2,6-$ dichlorophenolindophenol (DCIP) as the oxidant. In winter chloroplasts, as a mean of 5 measurements with two samples, 20 moles DCIP were photoreduced per mg chlorophyll per h at room temperature, whereas this figure for summer chloroplasts may even surpass 200. 


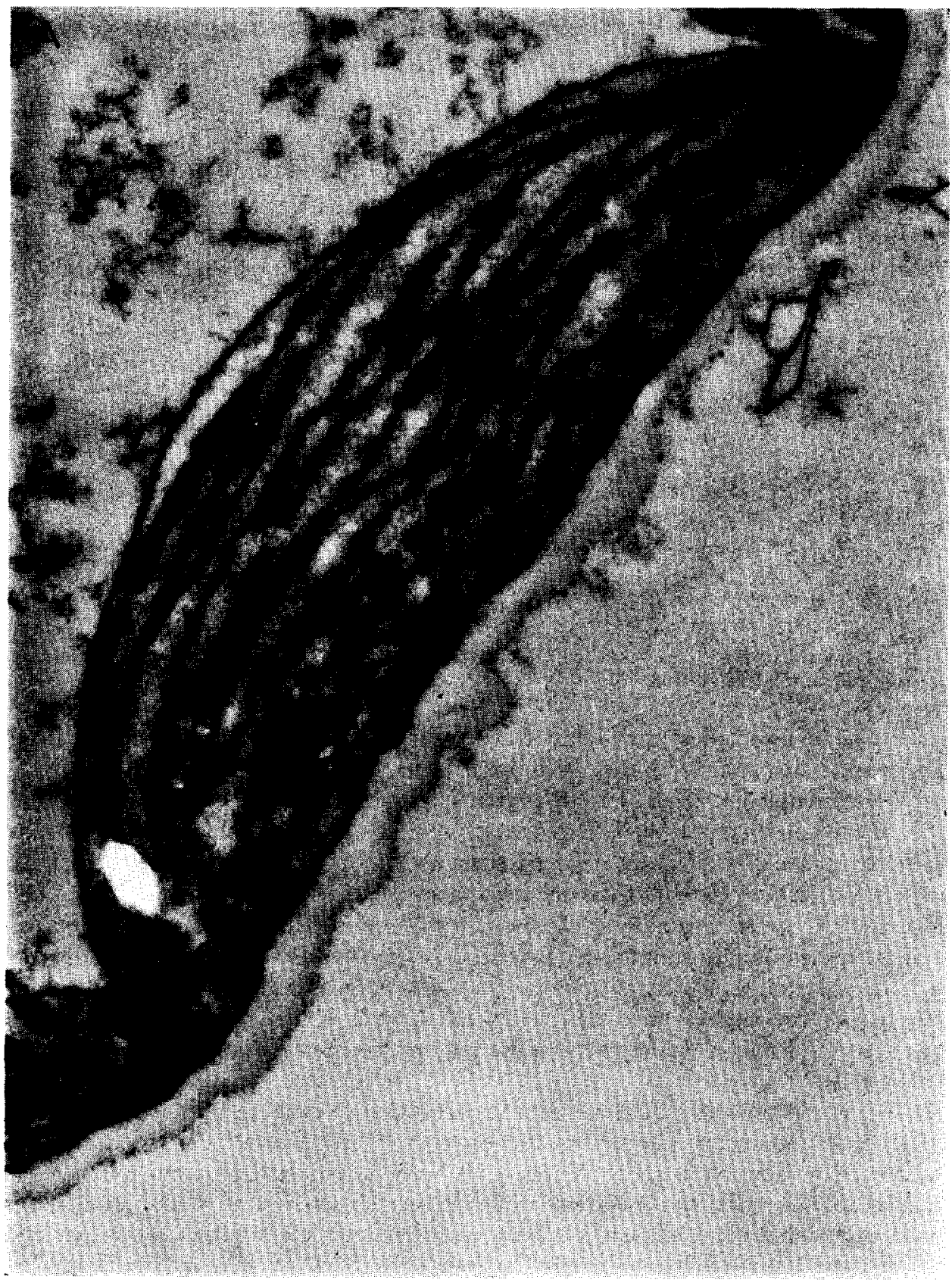

Fig. 3A. In situ chloroplast of spinach grown in summer. 


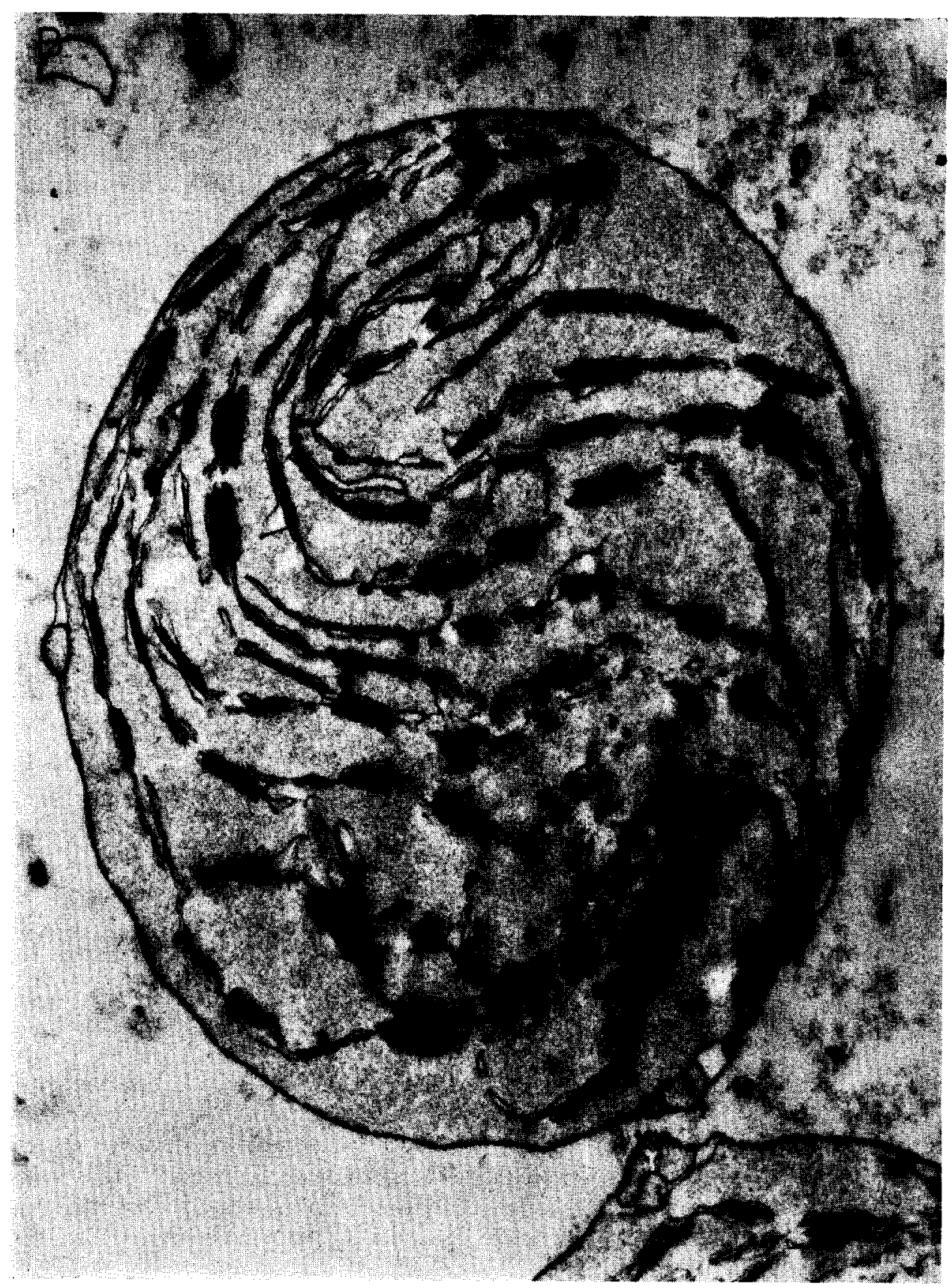

Fig. 3 B. In situ chloroplast of spinach grown in winter. 
In order to find out to what extent the dichroic effect is due to orientation of the chlorophyll molecules, dichroism was measured as a function of the concentration of sucrose added to the usual phosphate buffer, and, thus, as a function of the refractive index of the medium. The results are shown in Fig. 5. A clear minimum of the dichroic effect occurs at a sucrose concentration of about $0.18 \mathrm{M}$. The dichroic effect at this concentration is about $20 \%$ of that observed in the absence of sucrose. The minimum dichroism may occur for two reasons: (I) it may be due to orientation of chlorophyll molecules, and (2) it may still be of a textural origin if three instead of two layers of
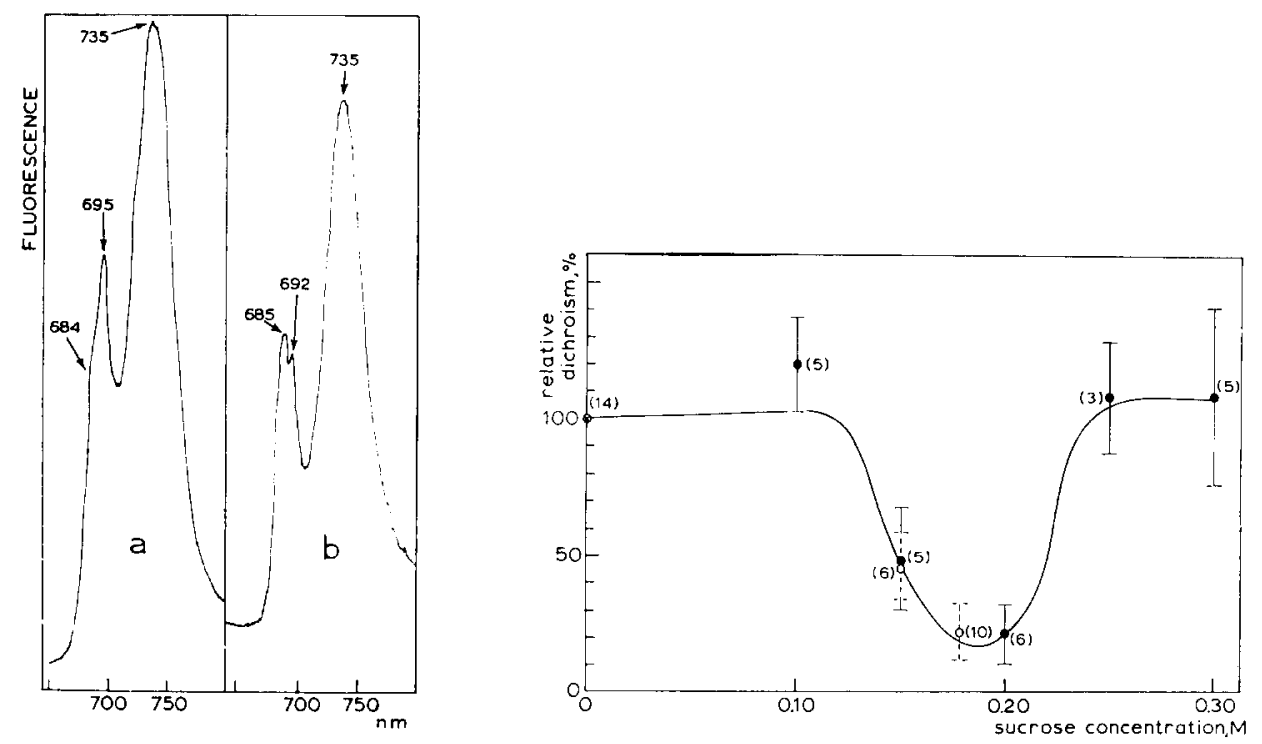

Fig. 4. Fluorescence spectra of summer chloroplasts (A) and winter chloroplasts (B) at liquid nitrogen temperature. The intensities of the two emission spectra are not comparable.

Fig. 5. Relative dichroism in oriented spinach chloroplasts as a function of sucrose concentration in the medium. The dichroic effect in the absence of sucrose is taken as roo \%. Bracketed figures indicate the number of experiments. Black dots with continuous vertical bars refer to mean percentages from I4 experiments, whereas open circles with broken vertical bars represent percentages for one and the same preparation. Vertical lines represent standard deviation of the mean.

different refractive indices are present. The occurrence of three such layers, namely a protein, a lipid, and a pigment layer, has been suggested by GOEDHEER ${ }^{6}$ when discussing his results on chlorophyll orientation in Mougeotia chloroplasts. However, if such were true in the present case, one would expect the shape of the graph in Fig. 5 to be asymmetric, or to show a shoulder in one of the descending parts, a second dip, or a broad minimum. Since the refractive index of such a suggested pigmented third layer is unknown, it is not possible to indicate at what particular sucrose concentration its effect may show up. Nevertheless, considering the type of electronic configuration of the chlorophyll molecule, one may expect the refractive index of a layer of chlorophyll-protein complexes to be in between those of proteins and lipids. Consequently, a second minimum considerably removed from the one shown in Fig. 5 is rather unlikely to occur. Since changes in textural dichroism due to anomalous dispersion are second-order effects, and hard to evaluate with the present technique, these are left 
out of consideration. Yet the symmetry of the graph and its narrow minimum suggest that no "third layer effect" shows up. The dichroism left at $0.18 \mathrm{M}$ sucrose, therefore, seems to be due to orientation of the chlorophyll molecules. Since (see above) in sucrose-free buffer the dichroic effect is of the order of $10 \%$, the results suggest that the dichroism due to chlorophyll orientation amounts to about $2 \%$, whereas the remaining, major, part should be considered to be textural dichroism. The dichroism due to oriented chlorophyll molecules in spinach chloroplasts is thus only rather weak, the dichroic ratio extinction $\|$ : extinction $\perp$ amounting to $1.02 \pm 0.005$ (representing the mean of $\mathrm{I}_{4}$ experiments). The orientation occurs in the plane of the lamellae. It might be expected that the dichroic effect at sucrose concentrations $0.10,0.25$, and $0.30 \mathrm{M}$ (Fig. 5) would be somewhat higher than without sucrose, since, in sucrose, the chloroplasts may tend to flatten due to osmotic phenomena. However, the scattering of the results is too great to justify such a conclusion.

\section{Checking the plane of maximum dichroism}

By rotating the system of two mutually perpendicularly transmitting polaroids in the described set-up, it was checked whether the dichroic effect is actually maximum in the plane of the lamellae. For textural dichroism such a relationship would be anticipated. However, since chlorophyll molecules might be arranged at a certain angle to the lamellae ${ }^{24}$, dichroism due to orientation of this pigment need not necessarily follow the same pattern. Therefore, the magnitude of the dichroic effect was determined as a function of the rotation of the polaroid system for chloroplasts in buffer without and with addition of sucrose up to a final concentration of $0.18 \mathrm{M}$.

Experiments without sucrose. The results of this series are shown in Fig. 6. It should be noted that the entire series of experiments was performed in early spring when the dichroic effect, viz. the values at $0^{\circ}\left(360^{\circ}\right)$ and $180^{\circ}$, was about half that observed in summer. Nevertheless, it is obvious that dichroism is maximum at $0^{\circ}$ $\left(360^{\circ}\right)$ and $180^{\circ}$, minimum at $90^{\circ}$ and $270^{\circ}$, and zero at $45^{\circ}, 135^{\circ}, 225^{\circ}$, and $315^{\circ}$. This relationship should indeed occur with textural dichroism inherent in the lamellar

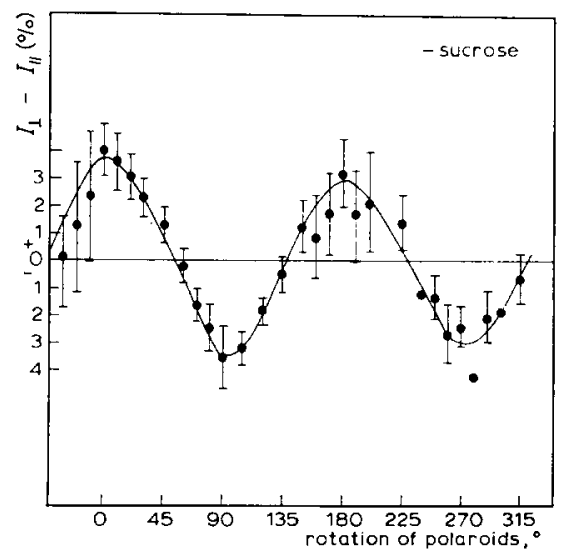

Fig. 6. Dichroic effect as a function of rotation of the polaroid system for spinach chloroplasts in buffer without sucrose. The points between $0^{\circ}$ and $135^{\circ}$, at $180^{\circ}, 270^{\circ}$ and $315^{\circ}$ each represent the means of 9 or Io experiments; at $\mathrm{I} 50^{\circ}$ and $225^{\circ}$ they refer to 8 experiments, whereas additional measurements of 4 experiments each were made for the remaining angles, except for $240^{\circ}, 280^{\circ}$ and $3^{\circ 0^{\circ}}$ where only 2 or 3 determinations were performed. 
structure of the chloroplasts. Since only about $\mathrm{I} / 5$ of the dichroic effect seems to originate from pigment orientation, the indicated errors do not allow any conclusion about a possible distortion in the shape of the curve (Fig. 6) due to a different relationship for the latter type of dichroism.

Experiments in the presence of $0.18 \mathrm{M}$ sucrose. The results are plotted in Fig. 7. As mentioned under METHODS, O.I8 $\mathrm{M}$ sucrose caused a prolonged decline of the dichroic effect as well as a decrease in the reproducibility of the measurements with time. In order to minimize distortion of the relationship in question, the series were measured from right to left and vice versa alternately. Such a procedure ensures that the decline does not show up in Fig. 7 , but it lowers the position of the curve relative to the original zero abscissa. The lowering of the values, therefore, is an artifact. We preferred to show the data without applying an estimated correction. The optical rotation inherent in a $0 . \mathrm{I} 8 \mathrm{M}$ sucrose solution amounts to $4.0^{\circ}$. It is thus sufficiently low so as not to influence the present results notably. In order to allow comparison, the graph was replotted in Fig. 8 (assuming that, according to expectation, the maximum and minimum are symmetric relative to the zero abscissa) together with a curve representing a calculated cosine function with an argument of twice the rotation angle of the polaroid system. Moreover, the mean of the errors from the graph in Fig. 7 is plotted as an area (between the thin dashed lines) surrounding the curve from the latter figure. It is clear that the reconstructed, experimental curve does not appreciably deviate from the theoretical one. That means that the experimental curve
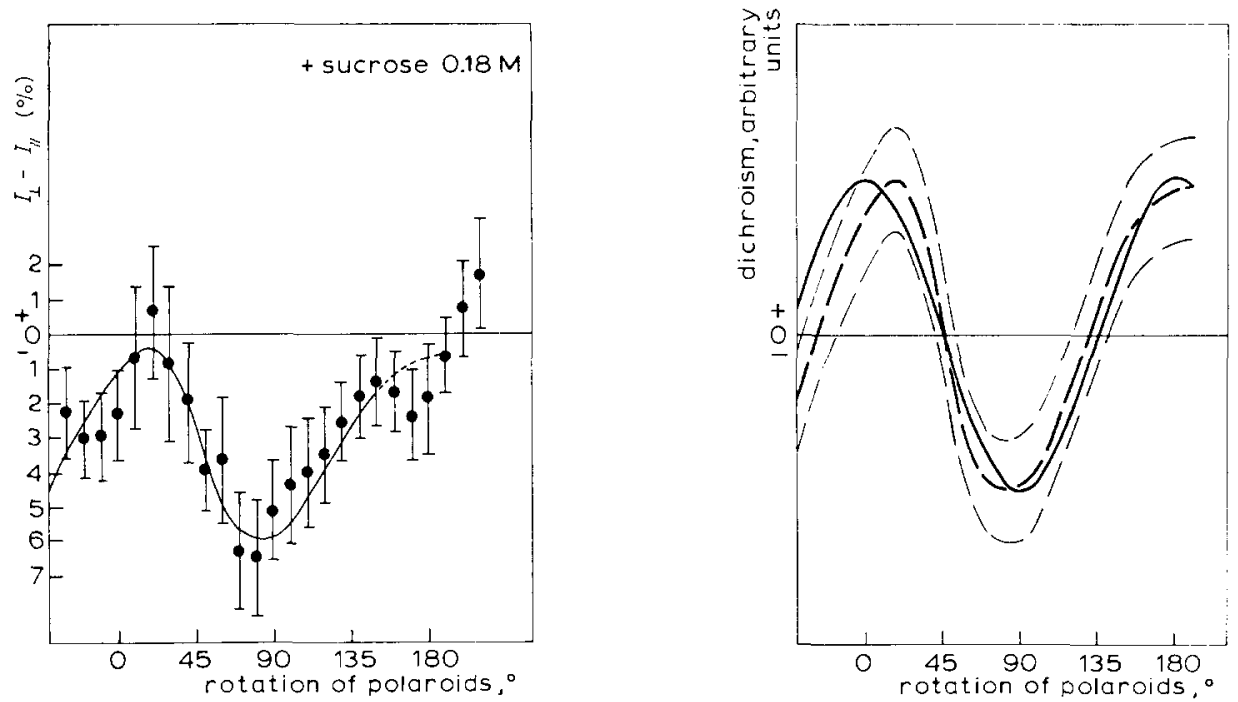

Fig. 7. Dichroic effect as a function of rotation of the polaroid system for spinach chloroplasts in buffer with $0.18 \mathrm{M}$ sucrose. The points at $170^{\circ}$ and $350^{\circ}$ represent means of 9 experiments, the others are each means of 10 experiments. The predominant negativity of the data is artificial (see text). Due to scattering of the results the shape of the curve beyond $135^{\circ}$ (broken line) is uncertain.

Fig. 8. Reconstructed graph from Fig. 7 (thick broken line) as well as a cosine function with an argument of twice the rotation angle of the polaroid system (continuous line). The thin broken lines represent the maximum and minimum boundaries of the errors of the means in Fig. 7. The "amplitudes" of the two curves are made to coincide, whereas the experimental graph is raised so as to become symmetrical relative to the zero abscissa. 
refers to a component oriented parallel to the steel-platelet surface. For, if this component were arranged at a certain angle relative to the mentioned surface, whereas in the experimental procedure used this angle were to be at random, the maxima and minima would be broadened in comparison with those of the theoretical curve. It is therefore concluded that no evidence is given for oriented chlorophyll molecules occupying positions other than in, or parallel to, the lamellar plane.

\section{Wavelength of maximum dichroism of orientation}

The absorption maximum of chlorophyll from spinach in vivo occurs around $679 \mathrm{~nm}$ (ref. 25). As illustrated by Fig. 2, the dichroic effect is maximum in the same region. However, the position of the red absorption maximum of chlorophyll $a$ in vivo is mainly determined by two chlorophyll types, $C_{a} 680$ and $C_{a} 670$. The maxima for both absorption and dichroism coincide only if both types contribute to the dichroic effect to the same extent as they do for absorption. It is therefore of interest to know whether these maxima actually coincide or not. Such a comparison makes sense only for chloroplasts in $0.18 \mathrm{M}$ sucrose-containing buffer. In 20 preparations the locations of the two peaks were compared. It was found that the dichroic maximum occurs at a slightly longer wavelength than that for absorption, the mean distance amounting to $0.90 \pm 0.30 \mathrm{~nm}$. However, since this value is quite close to the reproducibility inherent in the spectrophotometer used, it is given with reserve. Still, as the standard error indicates, there does seem to be a slight distance between the two maxima. In order to attempt an approximate estimation of the contribution of $C_{a} 680$ and $C_{a} 670$ to the dichroism of orientation, dichroism spectra were constructed assuming that (I) both chlorophyll types are present in the same amount, but contribute to dichroism to different degrees, (2) the absorption peaks are triangles with a half-width value of $20 \mathrm{~nm}$ and a top angle flattened by reducing the height by $5 \%$, and (3) the distance between the two maxima amounts to Io nm. According to these approximating conditions, it was found that $35 \%$ at most (say $1 / 3$ ) of the orientation dichroism may be due to the arrangement of $C_{a} 670$ complexes. Accepting this rough approximation and considering the result that about $2 \%$ of the total chlorophyll $a$ is oriented, it means that about $0.7 \%$ consists of oriented $C_{a} 670$ and about I.4\% of oriented $C_{a} 68 \mathrm{o}$. Or, putting it differently, of $C_{a} 670$ and $C_{a} 680$, about I.5\% (approx. $2 \times 0.7 \%$ ) and $3 \%$ (approx. $2 \times \mathrm{I} .4 \%$ ) respectively, occur in the oriented state.

\section{DISCUSSION}

The above experiments show that in "summer" chloroplasts from spinach a dichroic effect occurs. The results furthermore suggest that this effect is due to textural dichroism to an extent of $80 \%$, whereas the remainder, about $20 \%$, is likely to be ascribed to orientation of the porphyrin plates of chlorophyll $a$ complexes in a plane predominantly parallel to, or coinciding with, that of the lamellae. According to this suggestion, only about $2 \%$ of the total chlorophyll $a$ content is oriented. The dichroic ratio was found to be I.02. It was found by approximation that one-third, at the most, of the oriented complexes consists of the $C_{a} 670$ type, and two-thirds at least belongs to the $\mathrm{C}_{\boldsymbol{a}} 680$ type. In chloroplasts from spinach grown in winter without supplementary illumination, the dichroic effect due to structural changes did not show up.

Certain additional experiments indicate that, concomitantly, the Hill-reaction 
capacity as well as part of the energy transfer were reduced, again suggesting a relationship between structure and function.

Experiments on the effect of rotating the polaroid system on the orientation dichroism suggest that the plane in which the porphyrin plates of the chlorophyll molecules in question preferentially occur, is indeed parallel to that of the lamellae, rather than inclined to the latter at an angle smaller than $45^{\circ}$ or between $I 35^{\circ}$ and $180^{\circ}$. The present experiments reveal no orientation at all around $695 \mathrm{~nm}$. Instead they indicate a weak orientation around $680 \mathrm{~nm}$. The discrepancy with the results of SAUER AND CALVIN ${ }^{10}$ and SAUER ${ }^{14}$ can be readily explained by technical differences. These authors worked with quantasome suspensions, whereas quantasomes in situ are considered in the present study. Assuming that Sauer and Calvin oriented the quantasomes in a plane inclined at $45^{\circ}$ to the original plane of the lamellae, it follows that $\mathrm{C}_{\boldsymbol{a}} 695$ is oriented at an angle of $45^{\circ}$ towards the lamellae. This means that, in such a situation, they could not observe any dichroism at $680 \mathrm{~nm}$, whereas we were not able to find any dichroism at $695 \mathrm{~nm}$. Originally, Olson, Jennings And But$\mathrm{LER}^{8,9,11,12}$ concluded that $\mathrm{C}_{a} 695$ is oriented in the plane of the lamellae, but later, in accordance with the present results, BUTLER, OLSON AND JENNINGS ${ }^{13}$ concluded that it is $\mathrm{C}_{a} 680$ which is oriented in this plane.

Orientation of chlorophyll $b$ was not observed.

Starting from the above results and considerations, an attempt may be made to find a model for the pigment arrangement in the quantasome which also incorporates various data from the literature, and might reconcile some seemingly contradictory results. PARK AND BIGGINS ${ }^{20}$ concluded from electron-microscopical results that the quantasome, earlier ${ }^{26}$ believed to be the smallest unit in the photosynthetic apparatus, is composed of 4 or more subunits, and may occur in 3 types of packing. They measured the dimensions of the quantasome in chromium-shadowed spinach chloroplast lamellae to be I85 $\AA \times$ I55 $\AA \times$ Ioo $\AA$. Mühlethaler, Moor aNd SZARKOWSKI ${ }^{22}$ studied the same kind of chloroplasts using the freeze-etching technique $^{27}$ which enabled them to reduce deformations considerably. The latter authors concluded that spherical protein macromolecules occur on the inner and outer sides of the central layer (probably lipid) of the thylakoid membrane. On the outer side, the protein particles are arranged in groups of four ("multi-enzyme complexes"), whereas on the inner side the particles are scattered and less abundant. The groups of four are similar to the quantasomes of PARK AND BIGGINS. Each particle on both the inner and outer surface is actually spherical with a radius of $30 \AA$, and $20 \AA$ of it is embedded in the $40-\AA$-thick lipid layer.

By digitonin fragmentation WESSELS ${ }^{28}$ obtained subunits with a radius of $40 \AA$. These have a hollow centre and are attached by small stalks to the underlying membrane. The occurrence of such stalks was also reported by SiRonval et al. ${ }^{\mathbf{2 9}}$ in sonicated and digitoninized spinach preparations. Whether these stalks are artifacts due to digitionin or ultrasonic treatment remains to be studied. For the present purpose the presence or absence of stalks is not pertinent.

To avoid misinterpretation, mention should be made of quantasome-like structures found in blue-green ${ }^{30}$ and red algae; GANTT AND CONTI ${ }^{31}$ suggested that these structures, with a diameter of about $350 \AA$, are the sites of phycobilin concentration. Since their removal resulted in release of the phycobilins, but not of chlorophyll, and their dimensions are relatively large, it is clear that these structures are 
different from quantasomes. Actually, comparison of an electron micrograph made by these authors (their Fig. 7) with those of C-phycocyanin polymers made by BERNS AND EDWARDS $^{32}$ suggests that the particles in question represent clusters of phycobilin polymers.

WEIER AND BENSON ${ }^{33}$ discussed various hypotheses concerning the structure of the photosynthetic apparatus, and presented one themselves. The latter nicely includes various data from the literature as well. It emphasizes the structural role of a number of lipids, but the arrangement of chlorophylls and carotenoids is hard to reconcile with data on the orientation of these pigments. As the present results do not yield information about the chemical composition of the thylakoids, the following discussion will be restricted to considerations about orientation and will proceed from the submicroscopical scheme presented by MüHLethaleR ${ }^{21}$ and Mühlethaler, MOOR AND SZARKOWSKI ${ }^{22}$.

With this picture in mind, there are three possibilities for the location of the photosynthetic pigment molecules, namely, that they occur (I) in the spherical quantasome subunits, (2) in the underlying part of the lamellae, or (3) at both of these sites. Evidence has been obtained by various authors ( $c f$. e.g. refs. 34-38) demonstrating that chlorophyll in vivo is associated with proteins, or lipoproteins, rather than lipids. These data support the conception that the spherical subunits are carriers of the photosynthetic pigments.

PARK AND BIGGIN S $^{20}$ calculated that 230 chlorophyll molecules, when uniformly distributed, occur per quantasome. This number is low. Nevertheless it is more than twice that calculated by THOMAS, BLAAUW AND DUYSENS ${ }^{39}$ for particles from sonicated spinach leaves of a size critical for performing the Hill reaction. On the other hand, values of $300-400$ ( $c f$. refs. 40, 4I), 400-600 ( $c f$. ref. 42), or even higher ( $c f$. ref. 43) have been proposed by various authors. The cooperation of several quantasomes in photosynthesis has been suggested (e.g. ref. 42) and it may well be that such a combination accounts for the large chlorophyll numbers per photosynthetic unit. It should be borne in mind that the above-mentioned chlorophyll values, determined in various ways, are only approximate. In order to use intermediate values, the figures suggested by PARK AND BIGGINS ${ }^{20}$ will be considered in the further discussion.

The diameter of the quantasome subunits was estimated to be about Ioo $\AA$ by Park And Biggins ${ }^{20}$, and $60 \AA$ by MÜhlethaler, Moor and Szarkowski ${ }^{22}$. Since the former figure was derived from shadowed preparations, it is most probably somewhat exaggerated. BIGGINs AND PARK ${ }^{\mathbf{4 4}}$ suggested that 40 protein molecules, assumed to be spherical with a radius of $20 \AA$, occur per quantasome. They did not take into account the occurrence of scattered spherical particles located on the opposite side of the lamellae as found by MÜHLEThALER, MOOR AND SzARKowski ${ }^{22}$, and of the same size as the quantasome subunits. A rough approximation, deduced from the pictures by the latter authors, suggests that the scattered particles account for $40 \%$ of the total number. If this were true, it means that each quantasome subunit consists of 6 spherical protein molecules which jointly make up a sphere with a radius of $36 \AA$, and therefore a surface of about $\mathrm{I} 6000 \AA^{2}$. If one accepts the numbers for the pigment molecules in a spinach quantasome proposed by PARK AND BIGGINs ${ }^{20}$, a subunit carries 57 chlorophyll and 12 carotenoid molecules. If one then adds the volumes of these molecules to that of the protein sphere, the radius of such a combined particle amounts to $4 \mathrm{I} \hat{\AA}$. In such a sphere, quite close packing occurs. This would make it appear unlikely that 
the primary photoreactions of Systems I and 2 can proceed independently of each other when the pigments are located in the centre of the sphere. This conclusion bolds even if, as suggested by Cederstrand, Rabinowitch ANd GovindjeE ${ }^{45}$, the $\mathrm{C}_{a^{-}} 670$ and $\mathrm{C}_{a}$-680 chlorophyll types are distributed - albeit not evenly-over both systems. Spacing can only be obtained by arrangement of the pigment molecules in the surface region. We shall therefore consider the situation where the pigments occur on the surface of the subunits.

Since, in the present experiments, no indication has been obtained that the oscillators responsible for the red absorption - and, with them, the porphyrin plates as a whole are arranged otherwise than parallel to the lamellar plane, two situations remain to be discussed, namely (I) radial and (2) tangential arrangement relative to the subunit surface. Radial arrangement of the chlorophyll at the subunit surface would mean a $15-\AA$ increase in the radius if one disregards the phytol tails which may penetrate in between the proteins of the subunit. The radius of this subunit, then, would amount to $36+\mathrm{I} 5=5 \mathrm{I} \AA$. This dimension was actually measured by PARK AND BIGGINs ${ }^{20}$. However, as mentioned earlier, their value could be too large. Therefore, tangential arrangement of the chlorophyll molecules seems to be the preferred suggestion. The surface of a subunit with a $36-\AA$ radius is $16300 \AA^{2}$ and the combined areas occupied by 57 porphyrin plates and $\mathrm{I} 2$ "flat-lying" carotenoids amount to I $5600 \AA^{2}$. The closeness of these numbers does suggest that the pigment molecules are located on the outer side of the subunit surface.

The energy absorbed by the photosynthetic pigments is collected and fed into the chemical processes via trapping centres. $\mathrm{P} 700$ is supposed to act as such a centre for System I. KoK ${ }^{41}$ suggested that $\mathrm{C}$ 7oo functions merely as a trapping centre to pass its energy on to $\mathrm{P}$ 7oo. For the sake of simplicity the trapping centre will further be considered as a single complex: $\mathrm{P}$ 700. One $\mathrm{P} 700$, then, should be present in each quantasome. The same is suggested ${ }^{20}$ for the cytochromes $f$ and $b_{6}$, engaged in the mechanism of electron flow from the complex of cofactors indicated by $Q$. This means that these components are supposed to serve the four subunits of the quantasome, and, thus, are likely to be located in between these subunits. Considering the observation of Chance, Parsons AND Williams ${ }^{46}$ that cytochromes $b$ and $c$ in mitochondria are mainly located within the cristae, it is tempting to suggest that, by analogy, the cytochrome $f$ and $b_{\mathbf{6}}$ molecules of the quantasome may occur within the thylakoid near $Q$. The latter complex is possibly connected with a particle such as that observed by MÜhlethaler, MoOr and Szarkowski ${ }^{22}$ on the opposite side of the thylakoid. Such an arrangement is depicted in Fig. 9. The mutual location of the two cytochromes is given according to WITT et al. ${ }^{43}$. With the best fit, and contact with the System I $\mathrm{C}_{a}-680$ molecules in mind, the $\mathrm{P}-700$ molecule is placed in a vertical position. The concentration of $\mathrm{P} 700$ being rather low, no indication whatsoever about the situation of this complex was obtained from the dichroism measurements.

SaUer AND Calvin ${ }^{10}$ and SaUer ${ }^{14}$ concluded from electric and flow dichroism measurements in suspensions of quantasomes or quantasome aggregates that the $\mathrm{C}_{a}-695$ chlorophyll type is highly oriented. The concentration of $\mathrm{C}_{a} 695$ is estimated by these authors to be about $5 \%$ of that of the total chlorophyll. This means that in the scheme of Fig. 9, two $\mathrm{C}_{\boldsymbol{a}} 695$ occur per quantasome subunit. In view of the considerations given above, $\mathrm{C}_{a} 695$ is placed in our model at an angle of $45^{\circ}$ to the lamellae. The reason why this chlorophyll form is placed in the lower part of the subunits is to make 
the hypothesis of SAUER AND CALvin ${ }^{10}$ fit into the present picture. According to these authors, $\mathrm{C}_{\boldsymbol{a}} 695$ functions in reaction System 2. For reasons mentioned below, it is suggested that this system is located in the part of the subunits immersed in the $40-\AA$ lipid layer. SAUER AND CALvin hypothesize, moreover, that $C_{a} 695$ occurs close to a cytochrome molecule. Such a situation is hard to fit into the sort of scheme given in Fig. 9. It is therefore depicted in this figure in a more or less mirror-image location relative to its suggested position close to the centrally located cytochromes. In one respect, however, it is impossible to reconcile the present concept with that of SAUER And CALvin. The latter authors assume that about ro $\mathrm{C}_{a}-695$ molecules are highly oriented at a particular site, the quantatrope, thus influencing the absorption properties. According to our concept, this number is somewhat smaller, and the $8 C_{a}-695$ molecules are distributed over 4 subunits.

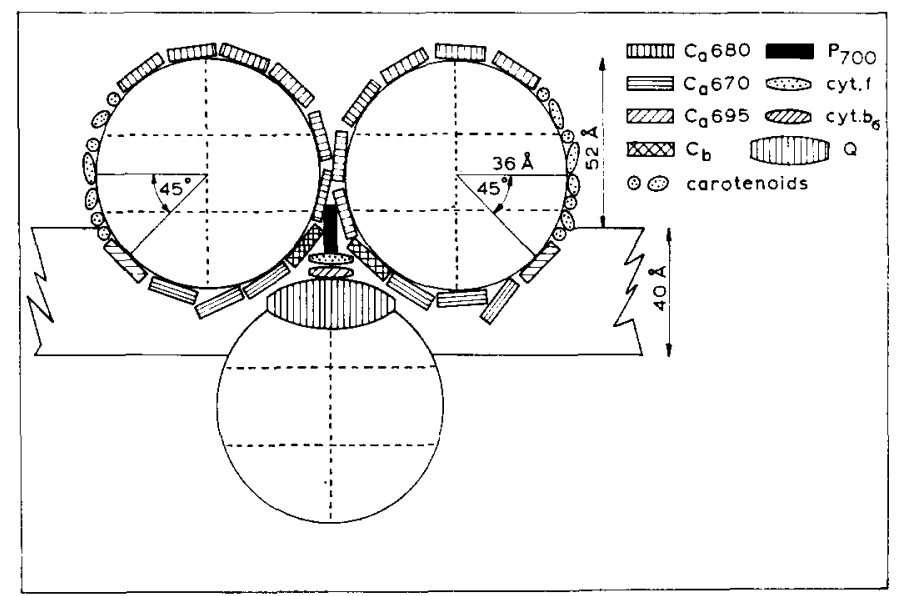

Fig. 9. Suggested arrangement in the spinach quantasome. Dotted lines indicate the presence of 6 protein molecules per subunit. According to Mühlethaler, Moor And Szarkowski ${ }^{22}$, the spherical particles are immersed up to $20 \AA$ in a 40 - $\AA$-thick lipid layer; the position of $C_{a} 695$ is indicated in a more or less mirror-image location. The phytol tails of the chlorophylls are not drawn. Of cytochromes $f$ and $b_{6}$ only the prosthetic groups are indicated. $Q$ represents a complex of cofactors which may be distributed over the entire particle. The diagram is a combination of two cross sections in parallel planes.

BOARDMAN AND ANDERSON ${ }^{\mathbf{4 7}}$ were the first to obtain (by digitonin fragmentation) chloroplast fractions which most likely contained the photochemical Systems I and 2. System $\mathrm{I}$ is believed to contain $\mathrm{C}_{a} 680$ as the major pigment, whereas that of System 2 is supposed to be $C_{a} 670$ ( $c f$. ref. 48 ). The particles carrying System I are much smaller than those incorporating System 2 (cf. ref. 49 ). For this reason $C_{a} 680$ is placed at the top of the model, whereas $C_{a} 670$ is situated on the subunit surface immersed in the lipid layer. Digitonin, then, is supposed to "dissect" the upper parts of the subunits leaving the lower parts in connection with each other via the lipid layer, thus forming larger particles than the $\mathrm{C}_{a}-680$ ones.

$\mathrm{C}_{\boldsymbol{a}} 68 \mathrm{o}$, tangentially arranged, cannot cover the entire upper half of the subunit sphere. Otherwise, and contrary to the present results, a preferential orientation perpendicular to the lamellar plane would be observed. Therefore, it must be located around the top part of the "upper" half of the subunits. In this way, however, no close 
proximity between $\mathrm{C}_{\boldsymbol{a}}-680$ molecules and $\mathrm{P} 700$ is obtained. Such a situation can, nevertheless, be realized by assuming that a short "tail" consisting of a few $\mathrm{C}_{\boldsymbol{a}}-68 \mathrm{o}$ molecules points "downwards" towards the centrally located P-70o complex. Such an arrangement, extending over an area resembling the wig of a British lawyer, makes the situation possible in which $2 \%$ of the total chlorophyll (according to the present suggestion) is oriented parallel to the lamellar plane. The $C_{a}-680$ "tail" must be suitably arranged to avoid the occurrence of preferential orientation perpendicular to the lamellar plane. Such is done in the model shown in Fig. Io.

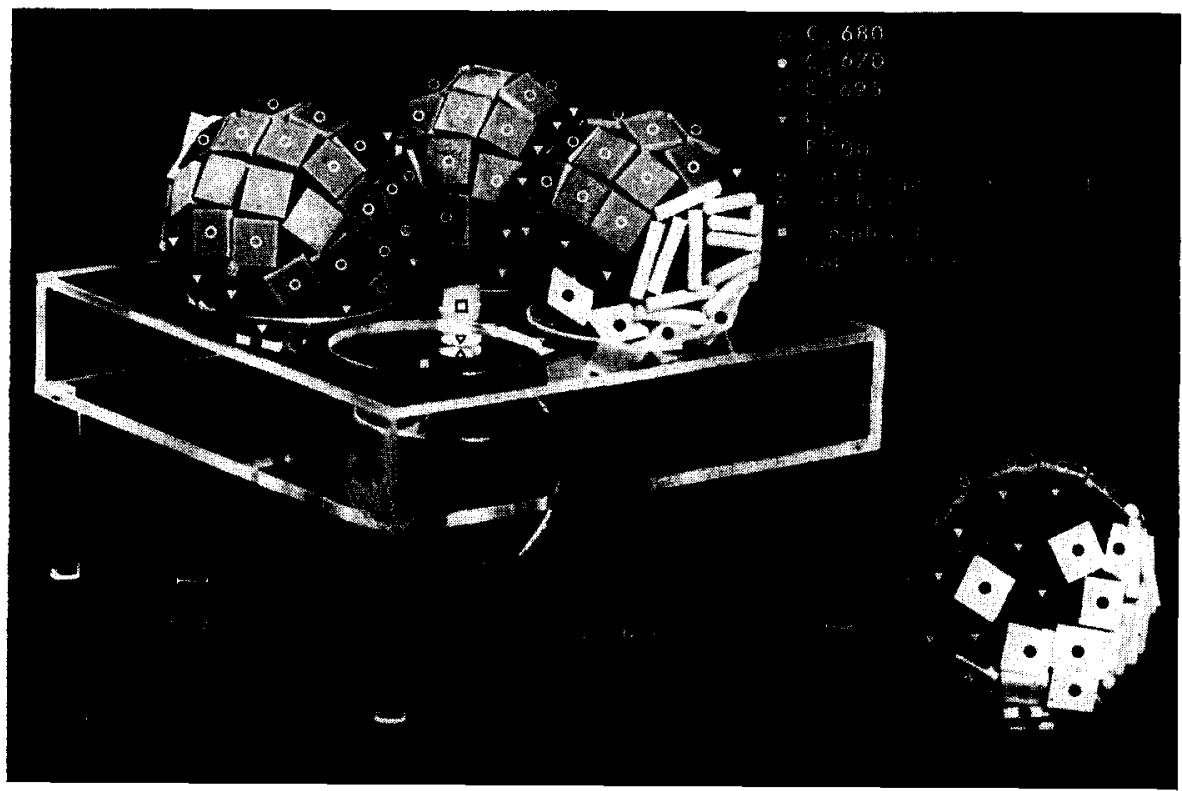

Fig. Io. Model of a spinach quantasome.

$\mathrm{C}_{a} 670$ cannot be located adjacent to $\mathrm{C}_{a} 680$, otherwise the latter type would act as an energy trap for $\mathrm{C}_{a} 670$. By bordering $\mathrm{C}_{a} 680$ with chlorophyll $b$ and carotenoid molecules, absorbing at shorter wavelengths than the chlorophyll $a$ types, an energy barrier between $C_{a} 670$ and $C_{a} 680$ is obtained. Since accessory pigments are shown to occur in both systems ${ }^{50}$, but to a larger extent in System 2 than in System $I$, the shape of the region occupied by these pigments is chosen such that the boundary between them and System 2 is longer than that with System I. In this way a higher probability for energy transfer from the accessory pigments to System 2 than to System I occurs. The resulting shape of the area occupied by chlorophyll $b$ is in accordance with the fact that no preferred orientation of this pigment is observed. The placing of the carotenoids in a more or less circular region on the opposite outer flanks of pairs of subunits in the quantasome is tentatively justified on the basis of the observation of MÜHLETHALER, MOOR AND SzARKowski ${ }^{22}$ (their Fig. II) that isolated quantasomes have a tendency to aggregate in linear, unbranched, arrays. Such an effect might mean that, at the sites of contact in the aggregates, the quantasomes display more or less hydrophobic properties that would account for their typical arrangement in suspension. The lipid character of carotenoids might account for this phenomenon. According to a suggestion by GOEDHEER ${ }^{6}$, these pigments may show a slight preference for orientation 
parallel to the lamellar plane. Our model, then, suggests that one of the functions of the accessory pigments may be the separation of Systems I and 2.

No indication of any intrinsic dichroism due to orientation of $\mathrm{C}_{a} 670$ was obtained by SAUER AND CALvin ${ }^{10}$ and SAUER ${ }^{14}$. The present experiments indicate that such a dichroism, if it occurs, is only rather weak. This conclusion agrees with the results of studies on fluorescence depolarization ${ }^{51,52}$. In accordance with what is said about the plane of orientation of the quantasomes, this would mean that the orientation of $C_{a} 670$ is nearly random. Such a situation can be obtained in the present model only if the tangential arrangement of these molecules on the subunit surface is imperfect. A model incorporating the present experimental results as well as the above considerations is shown in Fig. Io. It is emphatically stated that this model is hypothetical. It is only meant to contribute to the discussion and further analysis of the photosynthetic unit.

\section{ACKNOWLEDGEMENTS}

Thanks are due to Dr. P. F. ELBERS, Centre for Submicroscopical Study of Biological Objects, for carrying out the electron-microscopical work, to Dr. J. C. GoEDHEER for measuring fluorescence spectra, and to Mr. W. VERWER for determining Hill-reaction rates.

\section{REFERENCES}

I W. Menke, Kolloid- $Z$., 85 (I938) 256.

2 A. Frey-Wyssling and E. Steinmann, Biochim. Biophys. Acta, 2 (I948) 254.

3 W. Men Ke, Biol. Zentr., 63 (I943) 326.

4 W. Menke and G. MenKe, $Z$. Naturforsch., 106 (1955) 4 I6.

5 W. Menke, Z. Botan., 46 (I958) 26.

6 J. C. Goedheer, Ph. D. Thesis, State University, Utrecht, 1957.

7 F. Ruch, Exptl. Cell Res., Suppl., 4 (1957) 58.

8 R. A. Olson, W. L. Butler and W. H. Jennings, Biochim. Biophys. Acta, 54 (196I) 615.

9 R. A. Olson, W. L. Butler and W. H. Jennings, Biochim. Biophys. Acta, 58 (1962) I 44.

io K. Sauer and M. Calvin, J. Mol. Biol., 4 (I962) 45 I.

i I R. A. Olson, W. H. Jennings and W. L. Butler, Biochim. Biophys, Acta, 88 (1964) 318.

i2 R. A. Olson, W. H. Jennings and W. L. Butler, Biochim. Biophys. Acta, 88 (1964) 33 I.

I 3 W. L. Butler, R. A. Olson and W. H. Jennings, Biochim. Biophys. Acta, 88 (1964) 651.

I4 K. SAUER, Biophys. J,, 5 (1965) 337.

I 5 L. N. M. Duysens, Progr. Biophys, Biophys. Chem., I4 (1964) r.

i6 E. Katz, in J. Franck and W. E. Loomis, Photosynthesis in Plants, Iowa State College Press, Ames, 1949, p. 287.

I 7 J. A. Bassham and M. Calvin, U.S. At. Energy Comm., UCRL-2853 (1955).

I 8 W. Arnold and E. S. MeEk, Arch. Biochem. Biophys., 6o (1956) 809.

19 W. Arnold, J. Phys. Chem., 69 (1965) 788.

20 R. B. Park and J. Biggins, Science, I 44 (1964) roog.

2 I K. Mühlethaler, in T. W. Goodwin, Biochemistry of Chloroplasts, Vol. I, Academic Press, New York-London I966, p. 49.

22 K. Mühlethaler, H. Moor and J. W. Szarkowski, Planta, 67 (1965) 305.

23 J. B. Thomas, in C. Sironval, Le Chloroplaste, Masson, Paris, in the press.

24 E. A. Hanson, Rec. Trav. Botan. Neevl., 36 (I939) I83.

25 J. B. Thомаs, Biochim. Biophys, Acta, 59 (1962) 202.

26 R. B. Park and N. G. Pon, J. Mol. Biol., 3 (I96I) I.

27 H. Moor, Z. Zellforsch. Mikroskop. Anat., 62 (1964) 546.

28 J. S. C. Wessels, in J. B. Thomas and J. C. GoedheEr, Curvents in Photosynthesis, Donker, Rotterdam, I966, p. 129. 
29 C. Sironval, H. Clijsters, J.-M. Michel, R. Bronchart and R. M. Michel-Wolwertz, in J. B. Thomas and J. C. Goedhezr, Currents in Photosynthesis, Donker, Rotterdam, I966, p. II I.

3o M. Lefort, Compt. Rend., 26 I (I965) 223.

3 I E. Gantt and S. F. Contr, J. Cell Biol,, 29 (1966) 423.

32 D. S. Berns and M. R. Edwards, Arch. Biochem. Biophys., tio (I965) 5II.

33 T. E. Weier and A. A. Benson, in T. W. Goodwin, Biochemistry of Chloroplasts, Vol. I, Academic Press, New York-London, I966, p. 9 I .

34 D. W. Kupke, J. Biol. Chem., 237 (1962) 3287.

35 J. A. Gross, M. J. Becker and A. M. Shefner, Experientia, 20 (I964) $26 \mathrm{I}$.

36 P. Böger, Flora, I 54 (rg64) I 74 .

37 R. S. Criddle, in T. W. Goodwin, Biochemistry of Chloroplasts, Vol, I, Academic Press, New York-London, I966, p. 203.

$3^{8}$ J. B. Thomas and C. T. Bartels, in T. W. Goodwin, Biochemistry of Chloroplasts, Vol. I, Academic Press, New York-London, I966, p. 257.

39 J. B. Thomas, O. H. Blanuw and L. N. M. Duysens, Biochim. Biophys, Acta, Io (1953) 230. 40 B. KoK and G. Hoch, in W. D. McElroy and B. Glass, Light and Life, The Johns Hopkins Press, Baltimore, I96I, P. 397.

4 I B. Kok, in B. Kok AND A. T. Jagendorf, Photosynthetic Mechanisms of Green Planis, Publication No. I I 45, Natl. Acad. Sci.-Natl. Res. Council, Washington, I963, p. 45.

42 J. A. Gross, M. J. Becker and A. M. Shefner, Nature, 203 (I964) i 263.

43 H. 'T. Witt, B. Rumberg, P. Schmidt-Mende, U. Siggel, B. Skerra, J. Vater and J. We1Kard, Angew. Chem., 77 (1965) $82 \mathrm{I}$.

44 J. Biggins and R. B. Park, Plant Physiol., 40 (I965) i iog.

45 C. N. Cederstrand, E. Rabinowitch and Govindjee, Biochim. Biophys. Acta, i26 (I966) I.

46 B. Chance, D. F. Parsons and G. R. Williams, Science, i 43 (1964) 1 36.

47 N. K. Boardman and J. M. Anderson, Natuve, 203 (I964) I66.

48 J. H. C. Smith and C. S. French, Ann. Rev. Plant Physiol., I 4 (1963) г81.

49 C. N. Cederstrand and Govindjee, Biochim. Biophys. Acta, 120 (1966) I77.

50 L. N. M. Duysens and J. Amesz, Biochim. Biophys. Acta, 64 (1962) 243.

5 I J. Lavorel, Biochim. Biophys. Acta, 88 (1964) 20.

52 J. C. Goedheer, in T. W. Goodwin, Biochemistry of Chloroplasts, Vol. I, Academic Press, New York-London, I966, p. 75.

Biochim. Biophys. Acta, 143 (1967) 204-220 